\section{Economic Inequality Is Linked to Biased Self-Perception}

Psychological Science 22(10) I254-1258 (c) The Author(s) 2011 Reprints and permission: sagepub.com/journalsPermissions.nav DOI: $10.1177 / 095679761 / 417003$ http://pss.sagepub.com

@SAGE

\author{
Steve Loughnan ${ }^{1,2}$, Peter Kuppens ${ }^{1,3}$, Jüri Allik ${ }^{4,5}$, Katalin Balazs ${ }^{6}$, \\ Soledad de Lemus ${ }^{7}$, Kitty Dumont ${ }^{8}$, Rafael Gargurevich ${ }^{9}$, Istvan \\ Hidegkuti ${ }^{6}$, Bernhard Leidner ${ }^{10}$, Lennia Matos' ${ }^{9}$, Joonha Park ${ }^{1,11}$, \\ Anu Realo ${ }^{4}$, Junqi Shi ${ }^{12}$, Victor Eduardo Sojo ${ }^{13}$, Yuk-yue Tong ${ }^{14}$, \\ Jeroen Vaes ${ }^{15}$, Philippe Verduyn ${ }^{3}$, Victoria Yeung ${ }^{16}$, and \\ Nick Haslam ${ }^{\prime}$ \\ 'University of Melbourne; ${ }^{2}$ University of Kent; ${ }^{3}$ University of Leuven; ${ }^{4}$ University of Tartu; ${ }^{5}$ Estonian Academy of Sciences, \\ Tallinn, Estonia; ${ }^{6}$ University of Debrecen; ${ }^{7}$ Universidad de Granada; ${ }^{8}$ University of South Africa; ${ }^{9}$ Universidad Peruana \\ de Ciencias Aplicadas; ${ }^{10}$ University of Massachusetts, Amherst; "University of Tokyo; ${ }^{2}$ Peking University; \\ ${ }^{13}$ Universidad Central de Venezuela; ${ }^{14}$ Singapore Management University; ${ }^{15}$ University of Padova; and ${ }^{16}$ University of Hokkaido
}

\begin{abstract}
People's self-perception biases often lead them to see themselves as better than the average person (a phenomenon known as self-enhancement). This bias varies across cultures, and variations are typically explained using cultural variables, such as individualism versus collectivism. We propose that socioeconomic differences among societies-specifically, relative levels of economic inequality-play an important but unrecognized role in how people evaluate themselves. Evidence for selfenhancement was found in 15 diverse nations, but the magnitude of the bias varied. Greater self-enhancement was found in societies with more income inequality, and income inequality predicted cross-cultural differences in self-enhancement better than did individualism/collectivism. These results indicate that macrosocial differences in the distribution of economic goods are linked to microsocial processes of perceiving the self.
\end{abstract}

\title{
Keywords
}

self-perception, self-enhancement, income inequality, culture, self-esteem, sociocultural factors, socioeconomic status

Received I/5/II; Revision accepted 6/5/II

People often display a rosy bias in their self-perception. This self-enhancement bias is the tendency to emphasize or exaggerate one's desirable qualities relative to other people's (Alicke, 1985; Guenther \& Alicke, 2010). The magnitude of this bias varies across cultures, with people reporting higher levels of self-enhancement in some nations (e.g., the United States) than in others (e.g., Japan). These variations have been subject to extensive cross-cultural examination and debate centering on whether the desire to self-enhance is common to all people (Sedikides, Gaertner, \& Toguchi, 2003; Sedikides, Gaertner, \& Vevea, 2007) or stronger among Westerners, particularly North Americans, than among Easterners (Heine, 2003; Heine, Kitayama, \& Hamamura, 2007; Heine, Lehman, Markus, \& Kitayama, 1999). In a recent meta-analysis, Heine et al. (2007) found that 79 of 81 studies showed that Westerners were significantly more likely to self-enhance than Easterners.

The prevailing explanation of cross-cultural variability in levels of self-enhancement invokes the cultural dimensions of individualism and collectivism (Boucher, 2010; Chiu, Wan, Cheng, Kim, \& Yang, 2010; Triandis, 1995) and the associated concepts of independence and interdependence (Heine et al., 1999; Oyserman, Coon, \& Kemmelmeier, 2002). Theorists argue that Westerners are more likely to be individualists who seek personal success and uniqueness, and thus self-enhance more than do Easterners, who are more likely to be collectivists seeking interpersonal harmony and belonging (Boucher, 2010; Heine \& Hamamura, 2007). We propose an alternative explanation that favors socioeconomic differences over cultural dimensions. We suggest that the extent to which people engage in biased self-perception is influenced by the economic

\section{Corresponding Author:}

Steve Loughnan, University of Kent, Canterbury, Kent CT2 7NZ, United Kingdom

E-mail:s.loughnan@kent.ac.uk 
structure of their society, specifically its level of economic inequality.

Societies differ in their degree of economic inequality. In unequal societies, wealth is concentrated at the top, disadvantage at the bottom is extreme, and differences in social standing are highly salient (Kerbo, 2011; Wilkinson \& Pickett, 2010). Accordingly, in unequal societies, individuals are strongly motivated to stand out as superior to others. One expression of this desire may be to engage in stronger selfenhancement. In societies with more economic equality, the benefits of superiority diminish, and people's tendency to see themselves as above average should weaken. Indirect evidence that income inequality may be linked to self-enhancement comes from existing cross-cultural research. Japan and the United States-prototypical collectivist and individualist nations, respectively - also markedly differ on income equality. Among developed nations, Japan is one of the most equal and the United States is one of the least equal (Wilkinson \& Pickett, 2010). The reliable difference in levels of selfenhancement observed between these two nations may be a function not only of individualism versus collectivism, but also of covarying differences in each society's degree of economic equality.

Income inequality may foster greater self-enhancement through increased competition. Takata (2003) found that when Japanese participants were asked to compete over a limited resource under zero-sum conditions (i.e., the winner receives everything, the loser nothing), they displayed levels of selfenhancement similar to the levels displayed by Americans. That is, when people compete over concentrated rewards, they have a tendency to self-enhance. Because economic inequality polarizes benefits and costs and emphasizes hierarchy, people living in societies with high income inequality may see social relations as similar to zero-sum competitions. Thus, people in such societies should be particularly prone to self-enhancement, independent of levels of individualism.

The role of socioeconomic factors in understanding crosscultural differences has been increasingly recognized by researchers (Kitayama \& Uskul, 2011; Oyserman \& Lee, 2008). Demographic and economic differences between societies (e.g., population size, market integration) are predictive of social relationships. Socioeconomic differences explain cross-cultural variation in the extent to which people cooperate, exploit, and punish others (Henrich, Ensminger, et al., 2010; Marlowe et al., 2008). Although recent work has linked differences in social relations to socioeconomic factors, researchers have not examined how these differences might be related to perceptions of the self. We contributed to this emerging field by examining the association between economic inequality and biased self-perception.

In the study reported here, we investigated whether socioeconomic differences might be related to biased self-perception. Specifically, we expected economically unequal societies to be associated with an increased tendency to see the self as better than others (self-enhancement). We expected the effect of income inequality to be distinct from and more powerful than the effect of cultural differences in individualism and collectivism.

\section{Method}

We gathered data from 1,625 participants in five continents and 15 nations: Europe (Belgium, Estonia, Germany, Hungary, Italy, Spain), the Americas (Peru, the United States, Venezuela), Asia (China, Japan, Singapore, South Korea), Africa (South Africa), and Oceania (Australia). In 14 countries, participants were recruited from student populations at universities; in the United States, nonstudent participants were recruited online. Participants either volunteered, received course credit, or received payment. The samples varied in size ( $n=80-260$ per country), and participants were predominantly young (mean age $=21.55$ years, $S D=5.80$ years), with more females $(67 \%)$ than males $(33 \%)$.

Participants completed a standard questionnaire assessing self-enhancement. All questionnaires were translated into the native languages of the participants and back-translated for equivalence. The questionnaire asked participants to rate a set of personality traits and values on two dimensions. The first dimension, "How much do you possess this characteristic compared to the average student" (or "How much do you possess this characteristic compared to the average person" in the U.S. sample), was rated on a 7-point scale ranging from 1, much less than the average student/person, to 7, much more than the average student/person. The second dimension, "This characteristic is desirable, it is a characteristic that people generally want," was rated on a 7-point scale ranging from 1, not at all, to 7, very much so. The traits were selected on the basis of previous research to cover all domains of the Schwartz Value Survey (Schwartz, 1992) and the Big Five personality factors (Haslam, Bain, Douge, Lee, \& Bastian, 2005; Loughnan et al., 2010). For each language, four versions of the questionnaires were prepared. Participants randomly received one version. The questions on each version were identical, but the traits that participants were asked to rate differed. To reduce the amount of time required to complete the questionnaire, we included only 20 personality traits and values on each version, and this resulted in 80 personality traits and values being rated across the sample.

Societal indices were compiled from freely available sources. National levels of individualism, collectivism, and power distance (the extent to which people prefer an autocratic hierarchy versus a relative equality of power) were drawn from previous work (Hofstede, 2001). To assess economic inequality, we used national Gini coefficients, which gauge the income distribution within a society; as calculated by the United Nations, a Gini value of 100 means that a single individual receives all of the income (perfect inequality), and a Gini value of 0 means that income is evenly distributed across the population (perfect equality). Thus, higher values indicate greater inequality. Gini values were taken from the United 
Nations Human Development Report (United Nations Human Development Programme, 2010).

\section{Results}

Data were analyzed using multilevel modeling. Two-level multilevel regression models (with traits nested in persons) were estimated to obtain an average and separate random intercept and slope value for each nation. Three-level models (with traits nested in persons nested in nations) were estimated to examine the moderating role of income inequality and individualism/collectivism in self-enhancement. Both age and gender significantly moderated self-enhancement (age: $b=$ 0.003, $p=.044$; gender: $b=-0.05, p<.001$ ), and thus, all subsequent analyses controlled for these factors. Since 72 participants failed to report age or gender, the total number of participants was reduced to 1,553 individuals.

Self-enhancement was measured by regressing self-ratings onto trait desirability. This approach allows for individual and cultural differences independent of the relationship between self-ratings and desirability of traits. If people self-enhance, they should rate desirable qualities as especially self-descriptive relative to the average person (Guenther \& Alicke, 2010). In a two-level model predicting self-report from trait desirability and controlling for age and gender at the person level, a significant self-enhancement effect was found across nations using dummy-coded variables for each nation at the person level ( $b=0.24, p<.001)$. Further, a significant self-enhancement effect was found within every nation (all $p \mathrm{~s}<.022$ ). Consistent with the findings of prior research, our results showed that there was considerable variability between nations $(b \mathrm{~s}=$ 0.07-0.42).

Next, national Gini coefficients were included at the nation level in a three-level model. As income inequality increased, people's tendency to self-enhance also increased $(b=0.01, p=$ $.002)$. Next, we included individualism/collectivism alongside the Gini coefficients. In this model, income inequality remained a significant predictor of self-enhancement $(b=$ $0.01, p=.001$ ), and individualism did not significantly predict levels of self-enhancement above income inequality $(b<$ $-0.001, p=.331)$. Moreover, Gini coefficients were significantly better predictors of self-enhancement than individualism and collectivism were, $\chi^{2}(1)=25.40, p<.001$. A model with Gini coefficient and individualism/collectivism performed better than a model with individualism/collectivism only, $\chi^{2}(2)=15.94, p<.001$. By contrast, adding individualism/ collectivism to a model containing only Gini coefficients did not result in a significant improvement, $\chi^{2}(2)=0.05, p>.500$. These results indicate that, in this sample, income inequality was more important for explaining cross-national differences in levels of self-enhancement than individualism/collectivism were. For example, in comparing Venezuelans (high collectivism, high income inequality) with Japanese (high collectivism, low income inequality) in a two-level model with dummycoded nation variables, the former showed much higher levels of self-enhancement than did the latter ( $b=0.40$ vs. $b=0.07$, respectively).

We also investigated the influence of another cultural variable, power distance, which may be more closely linked to economic inequality than individualism and collectivism are. ${ }^{1}$ Power distance captures the extent to which people prefer an autocratic hierarchy versus a relative equality of power (Hofstede, 2001). When power-distance scores were included in the three-level model, Gini coefficients remained significant predictors of self-enhancement $(b=0.01, p=.001)$, but individualism/collectivism $(b<0.001, p=0.513)$ and power distance $(b<0.001, p=.986)$ did not.

There was a significant positive relationship between Gini coefficients and self-enhancement at the national level, $r(15)=$ $.79, p<.001$. As expected, people see themselves as superior to others to a greater extent in societies with a higher level of income inequality (Fig. 1). To investigate whether the observed positive prediction of self-rating by trait desirability reflected the belief that the self was indeed better than others, we selected the 20 most desirable traits in each nation. We then conducted a single-sample $t$ test for each nation to test whether the mean self-rating score for the 20 most desirable traits differed from the scale midpoint of 4 (which was labeled as neither less nor more than the average student/person). In 14 of the 15 nations, the average self-rating was indeed significantly higher than the scale midpoint for these desirable traits (all $p$ s <.01). Only Japanese participants failed to rate these traits significantly above the scale midpoint, $t(19)=1.36, p=.19$, although the mean was in the anticipated direction $(M=4.12)$. In short, people typically viewed themselves as better than average.

\section{Discussion}

The tendency for people to believe they possess more desirable characteristics than others appears widespread. It was observed in 15 nations spanning all developed continents. It is important to note that the magnitude of this self-enhancing bias varied according to societal differences in economic inequality. In societies with less income inequality, people showed a relatively weak bias compared with people in societies with more income inequality. It appears that people in societies with more income inequality tend to view themselves as superior to others, and people in societies with less income inequality tend to see themselves as more similar to their peers.

Socioeconomic differences accounted for the variability in this bias better than did individualism/collectivism, the cultural dimensions that have guided most previous research. This finding does not invalidate the utility of individualism/ collectivism for thinking about cultural differences. Indeed, these dimensions capture many East-West differences in selfand other perception, including self-enhancement (for recent reviews, see Heine \& Hamamura, 2007; Kitayama \& Uskul, 2011; Oyserman \& Lee, 2008). Our research emphasizes the importance of considering material differences between 


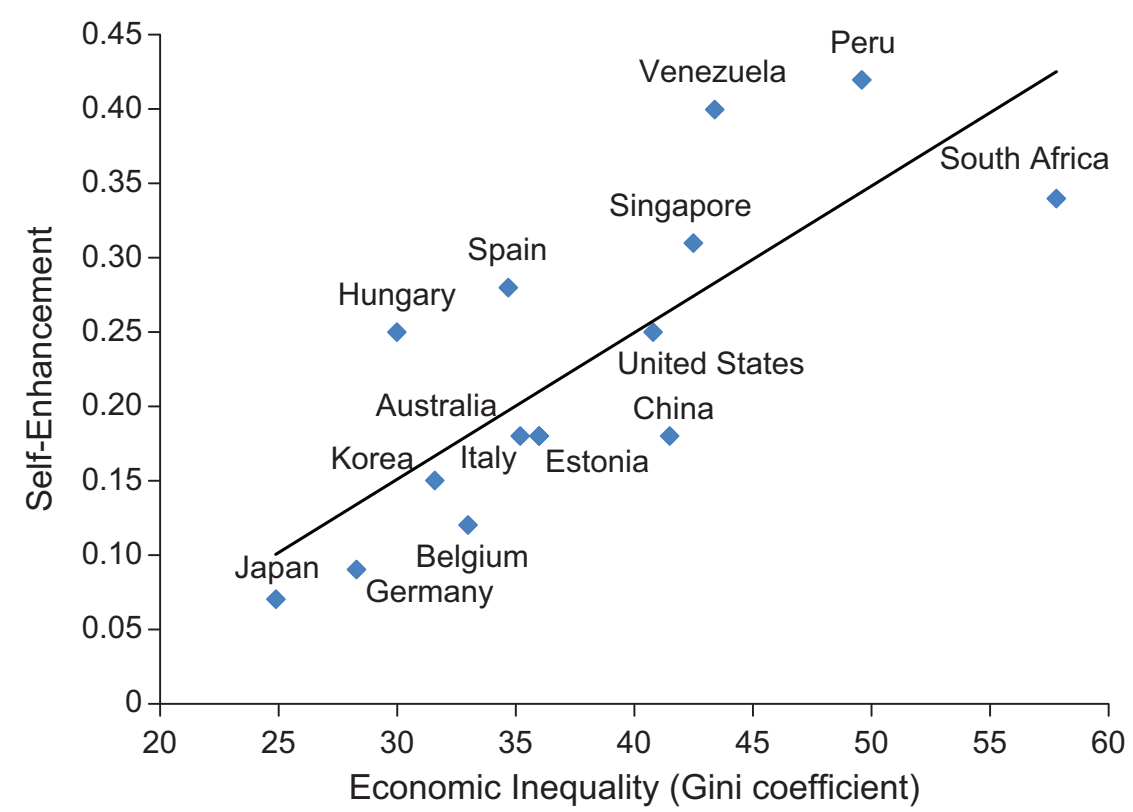

Fig. I. Scatter plot (with best-fitting regression line) showing self-enhancement (as indexed by beta weights from a two-level model) as a function of economic inequality (as indexed by the Gini coefficient) across nations. The data points for Australia and Italy are very close and overlap on the graph.

societies - particularly the distribution of income-when examining psychological differences. This emphasis is consistent with recent suggestions that people may respond to social and material conditions when self-enhancing (von Hippel \& Trivers, 2011).

It is unlikely that economic inequality directly leads to biased self-perception. It seems more likely that there are intervening factors that result from socioeconomic differences. One possibility raised earlier is perceived competition (Takata, 2003). When benefits and costs are polarized by inequality, people may compete for social superiority (Kerbo, 2011; Wilkinson \& Pickett, 2010). One manifestation of this drive may be the presentation of the self as superior through self-enhancement. Thus, it may be the competitiveness triggered by economic inequality that drives biased selfperception. It is interesting to note that competitiveness may be related to differences in individualism as well, with more individualistic societies also fostering greater competition (Chen \& West, 2008; Green, Deschamps, \& Paez, 2005). Both individualism and economic inequality may work in concert to foster a perception of competition that results in cultural differences in levels of self-enhancement. ${ }^{1}$ Likewise, both individualism and economic inequality may undermine the norm of modesty. Modesty norms play an important role in reducing self-enhancement, and when they are compromised, selfenhancement increases (Kurman, 2003, 2010). In societies with more income equality, people may not only have moreequal incomes, but they may also feel a pressure to seem more similar to others. This may manifest as a modesty norm, whereby people are discouraged from voicing both real and perceived superiority. Understanding the relationship between socioeconomic structure and individual psychology can help bridge the gulf between large-scale sociological studies of societies and individual social and psychological functioning.

An important limitation of our study should be noted. ${ }^{1}$ With the exception of the U.S. sample, our participants were drawn from university populations. Participant selection can seriously affect research findings (Henrich, Heine, \& Norenzayan, 2010), and our selection of university students might be confounded with levels of social inequality. University students might often find themselves in situations in which their social standing is actually better than the average person's, an effect which would be more pronounced in societies with more income inequality. Although we tried to guard against this confound by having participants compare themselves with the average student, a more robust test of whether economic inequality influences self-enhancement would involve sampling participants from groups receiving the median income in a society and examining their levels of self-enhancement.

In sum, cultural differences in self-perception have been the subject of extensive psychological research. The prevailing explanation of these differences has focused on the cultural dimensions of individualism and collectivism. In contrast, in the research reported in this article, we examined whether socioeconomic factors play a more important but unrecognized role in self-perception. It appears that aspects of macrolevel socioeconomic organization may be reflected in microlevel self-perception.

\section{Declaration of Conflicting Interests}

The authors declared that they had no conflicts of interest with respect to their authorship or the publication of this article. 


\section{Funding}

Steve Loughnan is a postdoctoral research associate funded by the Leverhulme Trust $(\mathrm{F} / 00236 / \mathrm{W})$. Peter Kuppens is a postdoctoral research fellow with the Fund for Scientific Research-Flanders and is supported by Katholieke Universiteit Leuven Research Council Grants GOA/05/04 and OT/11/031. Anu Realo and Jüri Allik were supported by a grant from the Estonian Ministry of Education and Science (SF0180029s08). Junqi Shi was supported by a grant from the National Nature Foundation of China (NSFC:71021001).

\section{Note}

1. We thank an anonymous reviewer for this suggestion.

\section{References}

Alicke, M. (1985). Global self-evaluation as determined by the desirability and controllability of trait adjectives. Journal of Personality and Social Psychology, 49, 1621-1630.

Boucher, H. (2010). Understanding Western-East Asian differences and similarities in self-enhancement. Social \& Personality Psychology Compass, 4, 304-317.

Chen, F., \& West, S. (2008). Measuring individualism and collectivism: The importance of considering differential components, reference groups, and measurement invariance. Journal of Research in Personality, 42, 259-294.

Chiu, C.-Y., Wan, C., Cheng, S., Kim, Y.-H., \& Yang, Y.-J. (2010). Cultural perspectives on self-enhancement and selfprotection. In M. Alicke \& C. Sedikides (Eds.), Handbook of selfenhancement and self-protection (pp. 425-446). New York, NY: Guilford Press.

Green, E., Deschamps, J., \& Paez, D. (2005). Variation of individualism and collectivism within and between 20 countries. Journal of Cross-Cultural Psychology, 36, 321-339.

Guenther, C., \& Alicke, M. (2010). Deconstructing the better-thanaverage effect. Journal of Personality and Social Psychology, 99, $755-770$.

Haslam, N., Bain, P., Douge, L., Lee, M., \& Bastian, B. (2005). More human than you: Attributing humanness to self and others. Journal of Personality and Social Psychology, 89, 937-950.

Heine, S. J. (2003). An exploration of cultural variation in selfenhancing and self-improving motivations. In V. Murphy-Berman \& J. Berman (Eds.), Nebraska Symposium on Motivation: Vol. 49. Cross-cultural differences in perspectives on the self (pp. 101-128). Lincoln: University of Nebraska Press.

Heine, S. J., \& Hamamura, T. (2007). In search of East Asian selfenhancement. Personality and Social Psychology Review, 11, 4-27.

Heine, S. J., Kitayama, S., \& Hamamura, T. (2007). Inclusion of additional studies yields different conclusions: Comment on Sedikides, Gaertner, \& Vevea (2005), Journal of Personality and Social Psychology. Asian Journal of Social Psychology, 10, 49-58.

Heine, S. J., Lehman, D., Markus, H., \& Kitayama, S. (1999). Is there a universal need for positive self-regard? Psychological Review, 106, 766-794.

Henrich, J., Ensminger, J., McElreath, R., Barr, A., Barrett, C., Bolyanatz, A., . . Z Ziker, J. (2010). Markets, religion, community size, and the evolution of fairness and punishment. Science, 327, $1480-1484$.

Henrich, J., Heine, S. J., \& Norenzayan, A. (2010). The weirdest people in the world? Behavioral \& Brain Sciences, 33, 61-83.

Hofstede, G. (2001). Culture's consequences: Comparing values, behaviors, institutions, and organizations across nations (2nd ed.). Thousand Oaks, CA: Sage.

Kerbo, H. (2011). Social stratification and inequality (8th ed.). New York, NY: McGraw-Hill.

Kitayama, S., \& Uskul, A. (2011). Culture, mind, and the brain: Current evidence and future directions. Annual Review of Psychology, 62, 419-449.

Kurman, J. (2003). Why is self-enhancement low in certain collectivistic cultures? An investigation of two competing explanations. Journal of Cross-Cultural Psychology, 34, 496-510.

Kurman, J. (2010). Good, better, best: Between culture and selfenhancement. Social \& Personality Psychology Compass, 4, 379 392.

Loughnan, S., Leidner, B., Doron, G., Haslam, N., Kashima, Y., Tong, J., \& Yeung, V. (2010). Universal biases in self-perception: Better and more human than average. British Journal of Social Psychology, 49, 627-636.

Marlowe, F., Berbesque, J. C., Barr, A., Barrett, C., Bolyanatz, A., Cardenas, J., . . . Tracer, D. (2008). More altruistic punishment in larger societies. Proceedings of the Royal Society B: Biological Sciences, 275, 587-590.

Oyserman, D., Coon, H., \& Kemmelmeier, M. (2002). Rethinking individualism and collectivism: Evaluation of theoretical assumptions and meta-analyses. Psychological Bulletin, 128, 3-72.

Oyserman, D., \& Lee, S. (2008). Does culture influence what and how we think? Effects of priming individualism and collectivism. Psychological Bulletin, 134, 311-342.

Schwartz, S. (1992). Universals in the content and structure of values: Theoretical advances and empirical tests in 20 countries. In M. P. Zanna (Ed.), Advances in experimental social psychology (Vol. 25, pp. 1-65). Orlando, FL: Academic Press.

Sedikides, C., Gaertner, L., \& Toguchi, Y. (2003). Pancultural selfenhancement. Journal of Personality and Social Psychology, 84, $60-79$.

Sedikides, C., Gaertner, L., \& Vevea, J. (2007). Evaluating the evidence for pancultural self-enhancement. Asian Journal of Social Psychology, 10, 201-203.

Takata, T. (2003). Self-enhancement and self-criticism in Japanese culture: An experimental analysis. Journal of Cross-Cultural Psychology, 34, 542-551.

Triandis, H. (1995). Individualism and collectivism. San Francisco, CA: Westview Press.

United Nations Human Development Programme. (2010). Human Development Report 2010. New York, NY: Author.

von Hippel, W., \& Trivers, R. (2011). The evolution and psychology of self-deception [Target article and commentary]. Behavioral \& Brain Sciences, 34, 1-56.

Wilkinson, R., \& Pickett, K. (2010). The spirit level: Why equality is better for everyone. London, England: Penguin. 